\title{
Die ökonomischen Kosten der fortbestehenden russischen Aggression gegen die Ukraine
}

https://doi.org/10.1515/sirius-2018-4004

Kurzfassung: Die Ukraine hat massive wirtschaftliche Verluste infolge der russischen Annexion der Krim im März 2014 und der militärischen Aggression gegen die östlichen Regionen Luhansk und Donetsk erleiden müssen. Während Russland auf der Krim einigermaßen funktionierende staatliche Strukturen eingeführt hat, ist die besetzte Donbass Region ein gesetzloses Dreckloch, aus dem mittlerweile die Hälfte der Bevölkerung geflohen ist. Russische Staatsbürger haben die Region geplündert und Unternehmen, Gebäude und Infrastruktur zerstört. Russische Handelssanktionen haben etwa 80 Prozent des Handels zwischen beiden Staaten eliminiert. Die Donbass-Region trug mit etwa 10 Prozent zum Bruttoinlandsprodukt der Ukraine bei, die Krim mit etwa 3,7\%. Wenn man von der Faustformel ausgeht, dass der Wert einer Region ungefähr dem Vierfachen des jährlichen Bruttoinlandsprodukts entspricht, so kann man den materiellen Verlust, der der Ukraine durch die russischen Aggressionen entstanden ist, bei etwa 98,4 Milliarden US Dollar ansetzen.

Schlüsselbegriffe: Ukraine, Russland, Krim, Kriegsschäden

Abstract: Ukraine has suffered great material losses because of the Russian annexation of Crimea in March 2014 and Russia's military aggression in the Ukrainian eastern regions of Luhansk and Donetsk since April 2014. Russia quickly introduced normal Russian rule in Crimea, while occupied Donbas is a lawless hellhole, from which half the population has fled. Russian subjects have seized assets and destroyed enterprises, buildings, and infrastructure. Russian trade sanctions have eliminated 80 percent of the mutual trade. The Donbas was the source of 10 percent of Ukraine's GDP and Crimea 3.7 percent. If we assess the total value of the assets Ukraine has lost at four times GDP, Ukraine has lost assets of a total of \$98.4 billion. Ukrainian subjects are suing the Russian Federation for compensation in several international courts.

Keywords: Russia, Ukraine, Crimea, war damage

\section{Einleitung}

Das Ziel dieser Analyse ist es, die materiellen Verluste abzuschätzen, die die Ukraine infolge der russischen Besetzung und Annexion der Krim im Februar/März 2014 sowie der militärischen Aggression Russlands in Teilen der ostukrainischen Gebiete Luhansk und Donezk seit 2014 erlitten hat. Diese beiden Fälle unterscheiden sich hinsichtlich der Form der militärischen Aggression deutlich voneinander, ebenso hinsichtlich der erlittenen Schäden und der Effizienz ihrer jeweiligen Verwaltungssysteme. Die Lebensverhältnisse in beiden Regionen sind schlecht, am schlechtesten im Donbass.

Es gilt dabei zwischen mehreren Arten von Kosten zu unterscheiden. Erstens wurden von der Regierung der Russischen Föderation beziehungsweise von Kräften, die von Russland unterstützt werden, Vermögenswerte beschlagnahmt. Zweitens kam es bei den Kampfhandlungen zur Zerstörung von Unternehmen, Gebäuden und Infrastruktureinrichtungen. Einige dieser Vermögenswerte gehörten dem ukrainischen Staat, andere Privatunternehmen oder -personen, aber bei allen handelt es sich um ukrainische juristische Personen. Eine weitere Kategorie von Verlusten sind Kapitalströme, die infolge von Sanktionen versiegt sind. Seit dem Sommer 2013 hat Russland immer strengere Handelssanktionen gegen die Ukraine verhängt, die schließlich ihrerseits mit eigenen Sanktionen antwortete. Außerdem verringerten sich infolge des Krieges die ausländischen Direktinvestitionen in der Ukraine.

In dieser Analyse geht es um die rein wirtschaftlichen Verluste, nicht um die militärischen oder humanitären Kosten. Daher wird das Thema der öffentlichen Ausgaben in den besetzten Gebieten nur gestreift, auch die Kosten von Binnenflüchtlingen werden nicht diskutiert. Die Untersuchung gelangt zu dem Schluss, dass die russische Aggression die Ukraine bislang etwa 100 Milliarden Dollar gekostet hat und dass der Verlust von Gas- und Erdölfeldern im Schwarzen Meer vor der Küste der Krim dabei in erheblichem Umfang zu Buche schlägt.

*Kontakt: Dr. Anders Åslund, Senior Fellow, Atlantic Council, Washington, DC, E-mail: AAslund@AtlanticCouncil.org 


\section{Chronik der Ereignisse}

Die russische Besetzung der Krim begann am 27. Februar 2014, als „grüne Männchen“ - russische Spezialkräfte ohne Hoheitszeichen - das Regionalparlament in Simferopol besetzten. Es war eine nicht provozierte militärische Aggression, die völlig überraschend kam. Die unvorbereiteten ukrainischen Streitkräfte leisteten keinen Widerstand, die Besetzung erfolgte praktisch ohne Blutvergießen. Am 18. März annektierte die Russische Föderation die gesamte Krim.

Im April 2014 versuchten „grüne Männchen“ in acht anderen Regionen im Süden und Osten der Ukraine unter dem Vorwand, dies sei eine lokale Erhebung russischsprachiger Ukrainer gegen die neue „illegitime“ Regierung in Kiew, kriegerische Auseinandersetzungen anzuzetteln. In Donezk und Luhansk waren sie damit erfolgreich. Erstaunlich schnell organisierten sich ukrainische Kräfte, darunter viele Freiwillige, und leisteten Widerstand. Im August zwang ihre Offensive den Kreml dazu, ein großes Kontingent regulärer russischer Truppen zu entsenden, um den Zusammenbruch der „Erhebungen“ zu verhindern.

Nach größeren russischen Offensiven wurden in Minsk zwei Waffenstillstandsvereinbarungen geschlossen. Die erste wurde am 5. September 2014 (zwei Wochen später folgte ein Memorandum) und die zweite am 12. Februar 2015 unterzeichnet. An den Verhandlungen nahmen Russland, die Ukraine, Frankreich, Deutschland und die Organisation für Sicherheit und Zusammenarbeit in Europa (OSZE) teil, aber die Vereinbarungen selbst unterzeichneten Vertreter der Ukraine und der sogenannten Volksrepubliken Donezk und Luhansk. Russland wird in dem Text nicht erwähnt; allerdings ist darin von „ausländischen Truppen“ die Rede, womit nach ukrainischer und westlicher Lesart russische Streitkräfte gemeint sind. Keines der beiden Waffenstillstandsabkommen wurde eingehalten, die Schusswechsel gehen täglich weiter und fordern immer wieder Todesopfer.

Seit September 2014 lautete die große Frage, ob russische Truppen versuchen würden, die bedeutende Industriestadt Mariupol zu erobern. Dies hätte verheerende Folgen für die ukrainische Wirtschaft, da Mariupol den zweitgrößten Hafen des Landes hat, über den ein Drittel der ukrainischen Exporte abgewickelt wird. Bislang ist das nicht geschehen. Ein Grund dafür mag sein, dass die wirtschaftlichen Schäden zu groß wären. Russland kontrolliert allerdings die schmale Straße von Kertsch, die ins Schwarze Meer führt, und hat zahlreiche Beschränkungen für den Schiffsverkehr von und nach Mariupol verhängt, sodass ein Großteil zu Häfen auf der anderen Seite der Krim umgeleitet wurde. Der Anteil der ukrainischen See- frachtexporte, die über die beiden ukrainischen Häfen am Asowschen Meer, Mariupol und Berdjansk, verschifft werden, sank von 16 Prozent in 2013 auf acht Prozent 2016. ${ }^{1}$

Die Ukraine hat die Zahl der militärischen und zivilen Todesopfer des Krieges veröffentlicht, sie übersteigt derzeit 10.300. Russland und seine subalternen Regime in Donezk und Luhansk machen keine Angaben über zivile oder militärische Verluste, aber es ist anzunehmen, dass auf der anderen Seite ähnlich viele Menschen getötet wurden. Zum Vergleich: Auf der Krim sind rund zwanzig Menschen verschwunden, mutmaßlich sechs von ihnen wurden getötet. Während dort eine strenge Diktatur herrscht, ist der Donbass eine gesetzlose Kriegszone.

Bevölkerungsbewegungen verdeutlichen den Gegensatz zwischen den beiden besetzten Gebieten. Die Krim hat, wie schon 2013, zwei Millionen Einwohner. Zwanzigtausend Menschen, hauptsächlich Krimtataren und Jugendliche, sind auf das ukrainische Festland ausgewandert und ungefähr genauso viele Russen zugewandert, die meisten davon vermutlich pensionierte Soldaten.

Dagegen fliehen die Menschen in Scharen aus dem Donbass. Zuverlässige Schätzungen sind schwer zu bekommen, aber ein umfangreicher Artikel, der im Juni 2015 vom russischen Medienunternehmen RBK veröffentlicht wurde (einer angesehenen Quelle unabhängiger Informationen und Recherchen, bevor der Kreml Druck auf es ausübte und es 2017 von einem Bekannten des russischen Präsidenten Vladimir Putin gekauft wurde), kam zu dem Ergebnis, dass die Gesamtbevölkerung von 6 bis 6,5 Millionen Einwohnern vor dem Krieg auf etwa drei Millionen gesunken sei. ${ }^{2}$ Nur etwa die Hälfte der Regionen Donezk und Luhansk sind besetzt, aber diese Gebiete schließen die beiden regionalen Hauptstädte und die meisten der Einwohner ein. Laut Schätzung von RBK leben 2 bis 2,5 Millionen davon in von Rebellen gehaltenen Gebieten und etwa eine Million von ihnen sind Rentner. Ein im Dezember 2016 in Bild erschienener Artikel veranschlagte die Zahl der Rentner in den besetzten Gebieten ebenfalls auf 1.079.000. ${ }^{3}$

Das ukrainische Sozialministerium berichtete im August 2016, 1.714.388 Personen seien in der Ukraine als Binnenflüchtlinge registriert ${ }^{4}$; dem RBK-Bericht zufolge

1 Saha/Kravchuk/Movchan 2018.

2 Ivan Golunov und Alexander Artemyev, „RBC Investigation: On Whose Money Does the Donbas Live?”, RBC, 15. Juni 2015, https:// www.rbc.ru/investigation/politics/15/06/2015/5579b4b99a79 $47 b 063440210$.

3 Julian Röpcke, „Milliarden aus Moskau. So finanziert der Kreml die ukrainischen Rebellen“, Bild-Zeitung, 16. Januar 2016.

4 UNHCR 2016. 
sind zwischen 500.000 und einer Million Menschen nach Russland ausgewandert und weitere 100.000 in andere Länder geflohen. Die Lebensverhältnisse im Donbass gestalten sich äußerst schwierig, geblieben sind fast nur Arme, Alte und Kriminelle.

Innerhalb des Donbass ist die Lage in Luhansk viel schlechter als in Donezk. Dort sind viele Bataillonskommandeure der Rebellen ermordet worden. Diese Morde wurden so gekonnt ausgeführt, dass der Verdacht naheliegt, es handele sich um gezielte Tötungen durch russische Spezialkräfte mit „Insiderwissen“. In Luhansk scheint nichts zu funktionieren oder produziert zu werden, während in Donezk ein gewisses Maß an Ordnung sowie eine elementare Lebensmittelversorgung und öffentliche Dienstleistungen wiederhergestellt wurden.

Das ist kein Vergleich zu den Verhältnissen auf der friedlichen und relativ gut organisierten Krim, die von Moskau nach russischem Gesetz und auf eine geordnete, wenn auch autoritäre Weise verwaltet wird. Die Beanstandungen auf der Krim betreffen die Diskriminierung von Krimtartaren und Ukrainern, Menschenrechtsverletzungen und Eigentumsdelikte. Es gibt aber keinen offenen Krieg oder gesetzlose Gangs wie im besetzten Donbass. Auch hat Russland die beiden besetzten Gebiete wirtschaftlich sehr unterschiedlich behandelt.

\section{Russische Politik auf der Krim}

Als Russland am 18. März 2014 die Krim annektierte, hat es die Region vollständig eingegliedert, das heißt russische staatliche Institutionen und Gesetze auferlegt, aber auch Finanzmittel der Russischen Föderation bereitgestellt. Der Rubel wurde eingeführt, ebenso russische Renten und Staatsgehälter. Es kam zur Enteignung des größten Teils der ukrainischen Privat- und Staatsunternehmen, während andere ukrainische Privatunternehmen gezwungen wurden, sich als russische Unternehmen registrieren $\mathrm{zu}$ lassen.

Die Vereinigten Staaten und die Europäische Union (EU) verhängten daraufhin einschneidende Sanktionen gegen russische Amtsträger, Privatpersonen und Unternehmen, die sie für die Annexion verantwortlich machten, sowie gegen jeden, der Geschäfte mit der Region betreibt. Die wirtschaftlichen Folgen sind gravierend. Der Außenhandel der Krim ist um 90 Prozent zurückgegangen. Die Immobilienpreise sind eingebrochen, während die Preise von Gütern und Dienstleistungen aufgrund von Versorgungsschwierigkeiten gestiegen sind. Im Tourismussektor ist die Zahl der Besucher von sechs Millionen pro
Jahr, zwei Drittel davon aus der Ukraine, auf vier bis fünf Millionen gesunken, die fast ausschließlich aus Russland kommen.

Die russische Regierung hat im Jahr 2014 etwa vier Milliarden Dollar in die zivile Wirtschaft der Krim gepumpt und subventioniert die Region weiterhin mit etwa zwei Milliarden Dollar pro Jahr. Sie baut für rund 3,5 Milliarden Dollar eine Brücke über die Straße von Kertsch, die das russische Festland mit der Ostspitze der Krim verbindet und die im Mai 2018 teileröffnet worden ist. Das Projekt wird von Arkadi Rotenberg geleitet, einem Baumagnaten und langjährigen Freund Putins, der persönlich ebenso von Sanktionen betroffen ist wie viele seiner Unternehmen, die am Bau der Brücke beteiligt sind. ${ }^{5}$

Die Wirtschaft der Krim ist mittlerweile vollkommen abhängig vom russischen Staat und wird es wahrscheinlich bleiben; die jährlichen Kosten von zwei Milliarden Dollar, lediglich 0,14 Prozent des gegenwärtigen russischen BIP von 1,4 Billionen Dollar, sind zwar nicht unerheblich, aber für Russland zu verkraften. Die militärischen Ausgaben für die Krim sind darin allerdings nicht enthalten.

\section{Materielle Verluste der Ukraine infolge der Krim-Annexion}

Die größten materiellen Verluste der Ukraine auf der Krim betreffen Land, Energieressourcen, Banken mitsamt ihren Vermögenswerten, Unternehmen und Immobilien. Mit der Annexion durch Russland verlor die Ukraine dort sofort ihr gesamtes staatliches Eigentum, zu dem Naftogaz-Beteiligungen, Infrastruktur, Gebäude, Land und militärische Ressourcen gehörten.

Die größten Verluste betrafen Gasvorkommen. Von der ukrainischen Erdgasproduktion von 20 Milliarden Kubikmeter pro Jahr kamen 1,6 Milliarden Kubikmeter von dem Staatsunternehmen Chornomornaftogaz auf der Krim, das 18 Gasfelder an Land und im Schwarzmeerschelf ausbeutete. ${ }^{6}$ Das Unternehmen wurde im März 2014 von den Regionalbehörden der Krim ,verstaatlicht“ und befin-

5 US Department of the Treasury, „Treasury Sanctions Russian Officials, Members Of The Russian Leadership's Inner Circle, And An Entity For Involvement In The Situation In Ukraine”, Pressemitteilung vom 20. März 2014, https://www.treasury.gov/press-center/ press-releases/Pages/j123331.aspx, und US Department of the Treasur, „Treasury Sanctions Individuals and Entities for Sanctions Evasion and Activities Related to the Conflict in Ukraine”, Pressemitteilung vom 1. September 2016, https://www.treasury.gov/press-center/ press-releases/Pages/j15048.aspx.

6 Bentzen 2015. 
det sich gegenwärtig faktisch unter russischer Kontrolle. Maksym Bugriy, ein ukrainischer Volkswirt und Wertpapieranalyst, wies kurz nach der Annexion darauf hin, Chornomornaftogaz habe die Produktion im Jahr 2015 verdoppeln wollen. Davon ausgehend veranschlagte er den Wert des Unternehmens auf eine Milliarde Dollar, eine recht niedrige Schätzung. ${ }^{7}$

Die Ukraine verfügt über große, unerschlossene Gasvorkommen in ihrem Schelf im Schwarzen Meer und im Asowschem Meer. Mit der Annexion hat Russland auch diese Vorkommen in Besitz genommen. ${ }^{8}$ Im April 2014 erklärte der ukrainische Energieminister Yuriy Prodan, der Verlust des Zugangs zu den Feldern im Schwarzen Meer werde das Land 40 Milliarden Dollar kosten, aber damals waren die Erdöl- und Erdgaspreise noch immer sehr hoch.

An dem zuvor bedeutendsten dieser Offshore-Projekte war ein Konsortium unter Führung von ExxonMobil beteiligt, dem auch Royal Dutch Shell und die rumänische OMV Petrom angehörten. Vorangetrieben werden sollten damit Production-Sharing Agreements (PSAs), die seit 1999 gesetzlich verankert sind, für Offshore-Felder außerhalb der Krim. Diese Pläne wurden wegen der Annexion und der anschließenden westlichen Sanktionen gegen Russland und die Krim zurückgestellt. Bugriy schätzte den Wert der Reserven allein in diesem Feld auf 30 Milliarden Dollar, sofern der Gaspreis weiterhin hoch bliebe. ${ }^{9}$

Seit der Annexion hat Russland zudem in großem Umfang öffentliches und privates Eigentum beschlagnahmt und diese Maßnahmen als "Verstaatlichungen“ gemäß den Gesetzen der Russischen Föderation bezeichnet. Per 12. Mai 2017 sind 4.575 öffentliche und private Immobilien verstaatlicht worden. Außerdem haben russische Behörden den Abriss von Gebäuden erlaubt, die ohne die erforderlichen Genehmigungen errichtet worden waren. Die russischen Behörden haben Schritte unternommen, um die Eigentümer beschlagnahmter Immobilien zu entschädigen, aber Personen, denen „Extremismus“ vorgeworfen wird, sind davon ausgenommen. Außerdem können Entschädigungszahlungen zehn Jahre lang hinausgeschoben werden. ${ }^{10} \mathrm{Im}$ Sommer 2017 wurden in einem Zeitraum von drei Monaten 600 private Liegenschaften in Sewastopol übernommen, wobei die Alteigentümer möglicherweise keine Entschädigung erhalten. Mehrere Immobilienbesitzer mussten erleben, dass Gerichte Kaufverträge, die vor der russischen Besetzung geschlossen worden waren, aufhoben; diese gerichtlichen

7 Bugriy 2014.

8 BP 2014.

9 Bugriy 2014.

10 UNHCR 2017a.
Entscheidungen laufen auf eine entschädigungslose Enteignung hinaus. ${ }^{11}$

Dem ukrainischen Staat gehörten auch 1,4 Millionen Hektar Agrarland auf der Krim. ${ }^{12}$ Der gegenwärtige Durchschnittspreis für Agrarland in der Ukraine beträgt 1.300 Dollar pro Hektar, was einem Gesamtwert von 1,8 Milliarden Dollar entspräche. Käme es zur Liberalisierung des Grundstücksmarktes und legt man den Grundstückspreis in benachbarten EU-Ländern zugrunde, würde der Preis wahrscheinlich auf 4.000 Dollar pro Hektar steigen. Dann wäre dieser Grundbesitz 5,6 Milliarden Dollar wert.

Privatunternehmen auf der Krim sehen sich seit der Annexion vor die Wahl gestellt, sich entweder als russische Unternehmen neu registrieren zu lassen oder enteignet zu werden. Entscheiden sie sich für Ersteres, fallen sie unter die strengen westlichen Sanktionen gegen die Krim, die im März 2014 verhängt worden sind. Viele ukrainische Eigentümer haben versucht, ihre Immobilien auf der Krim zu verkaufen, aber die anhaltende Marktflaute und die niedrigen Preise halten sie davon $a b .{ }^{13}$ Insgesamt sind über 400 ukrainische Unternehmen enteignet worden. ${ }^{14}$ Die beiden größten Unternehmen auf der Krim, Crimea Soda und Crimea Titan, die dem ukrainischen Oligarchen Dmytro Firtaš gehören, wurden offenbar als russische Unternehmen neu registriert. ${ }^{15}$

Die Krim hatte einen großen Bankensektor. Im Februar 2014 waren dort über zwanzig ukrainische Banken mit 1.022 Zweigstellen vertreten. Die Nationalbank der Ukraine schätzte den Wert des regionalen Bankensektors auf 1,7 bis 1,9 Milliarden Dollar. ${ }^{16}$ Ende April hatten alle in der Ukraine registrierten Banken ihre Filialen geschlossen und die Krim verlassen. Die größten russischen Staatsbanken, Sberbank und VTB, blieben weg, um den Sanktionen der USA und der EU zu entgehen. Dafür eröffneten die bereits sanktionierte Bank Rossiya, die Freunden Putins in St. Petersburg gehört, und einige kleinere russische Staatsbanken, insbesondere die Russian National Commercial

\section{Ebenda.}

12 Bugriy 2014.

13 Gespräche mit drei Immobilienbesitzern auf der Krim.

14 Bentzen 2015.

15 Vgl. die „Geschichte“ von Crim Soda, http://www.sodaplant.ru/ en/about/history/.

16 Steve Stecklow/Elizabeth Piper/Oleksandr Akymenko, „Special Report: Crimean savers ask: Where's our money?”, Reuters, 20. November 2014, https://www.reuters.com/article/us-ukraine-crisisbanks-specialreport/special-report-crimean-savers-ask-wheresour-moneyidUSKCNOJ40FJ20141120?irpc=932; vgl. auch „Ukrainian Banks Quit Crimea”, RT, 15. April 2014, https://www.rt.com/business/ ukraine-banksclose-crimea-572/. 
Bank (RNCB), Filialen auf der Krim. ${ }^{17}$ Diese hat sich als die russische Staatsbank für Regionen mit eingefrorenen Konflikten etabliert.

\section{Russische Politik im Donbass}

Während die russische Regierung auf der Krim einem eindeutigen Kurs der Annexion und Verstaatlichung folgt, ist ihre Politik im Donbass weder klar noch beständig. Da sie beharrlich jegliche Präsenz dort bestreitet, hat die russische Regierung keine offizielle Verantwortung für Verwaltung oder Wirtschaft übernommen. Dieses Vorgehen hat dazu geführt, dass die Region in einem gesetzlosen Chaos versunken ist.

In der zweiten Hälfte des Jahres 2014 kam es im besetzten Donbass zu schrecklichen Zerstörungen. Russische Pioniertruppen sprengten Brücken und Fabriken waren heftigem Artilleriebeschuss ausgesetzt. Alle Banken und Geldautomaten wurden geplündert und die Bankgeschäfte eingestellt. Die meisten Läden schlossen, sodass praktisch nur noch Lebensmittelgeschäfte übrigblieben. Rebellen plünderten oder beschlagnahmten kleine, mittlere und staatliche Unternehmen, während private Großunternehmen, die weitgehend von Rinat Achmetovs Firmen DTEK und Metinvest kontrolliert wurden, weiterarbeiten durften. ${ }^{18}$ Sie waren dort weiterhin ebenso wie auf ukrainischem Territorium tätig und ihre Beschäftigten mussten ihre Löhne an Geldautomaten in der freien Ukraine abheben. ${ }^{19}$ Die ersten Anzeichen einer Stabilisierung zeigten sich im August 2014, als Alexander Zacharčenko Ministerpräsident der Volksrepublik Donezk wurde und Igor Plotnickij Oberhaupt der Volksrepublik Luhansk. Zacharčenko war bis zu seinem Tod im August 2018 nomineller Anführer der Volksrepublik Donezk, Plotnicki wurde im November 2017 bei einem Coup gestürzt und von Leonid Pasechnik abgelöst. Diese Führer gelten als mittelmäßig und uncharismatisch, aber sie sind Moskau hörig.

17 Alissa de Carbonnel und Elizabeth Piper, „Sanctions Trump Patriotism for Russian Banks in Crimea”, Reuters, 9. April 2014, https://www. reuters.com/article/ukraine-crisis-crimea-banks/sanctions-trumppatriotism-for-russian-banks-in-crimea-idUSL6NON01X620140409.

18 Anders Åslund, „New Russian Management of the Donbas Signifies Putin May Be Ready to Negotiate”, Atlantic Council, 4. Januar 2016.

19 Pavel Kanygin, „The Donbass War. Assessing the Aftermath: How the 'Russian Spring' Came to an End in Eastern Ukraine”, Meduza, 13. November 2015, https://meduza.io/en/feature/2015/11/13/thedonbass-war-assessing-the-aftermath; Anders Åslund, „New Russian Management of the Donbas Signifies Putin May Be Ready to Negotiate”.
Kiew zahlte bis November 2014 weiterhin Renten im Donbass. Dann unterzeichnete Präsident Petro Porošenko ein Dekret, das die besetzten Gebiete von sämtlichen ukrainischen Staatsgeldern abschnitt. Die Ukraine konnte sich keine großen öffentlichen Ausgaben für Gebiete leisten, die sie nicht kontrollierte und in denen sie keine Steuern erheben konnte. Während der Minsker Verhandlungen im Februar 2015 forderte Russland, die ukrainische Regierung solle weiterhin öffentliche Mittel für den besetzten Donbass bereitstellen, aber diese zahlte Renten nur an Personen aus, die ihren Wohnsitz in den nicht-besetzten Gebieten des Landes anmeldeten. ${ }^{20}$ Im April 2015 begann Russland zögerlich damit, sporadisch Renten im Donbass auszuzahlen und einige grundlegende öffentliche Ausgaben in Rubel abzudecken. Im September 2015 erklärte die Volksrepublik Luhansk den Rubel zu ihrem offiziellen Zahlungsmittel und die Volksrepublik Donezk zog im Oktober nach. ${ }^{21}$ Bis November 2015 scheint sich die Lage so dramatisch verschlechtert zu haben, dass Moskau eine Hungersnot befürchtete und begann, regelmäßig Renten und staatliche Gehälter allerdings in minimaler Höhe auszuzahlen. Die International Crisis Group veranschlagt die Kosten dieser Zahlungen für Russland auf eine Milliarde Dollar pro Jahr. ${ }^{22}$ Nach einer glaubwürdigen Schätzung der ukrainischen Regierung hat Moskau 2016 in den von den Rebellen gehaltenen Gebieten zwei Milliarden Dollar für nichtmilitärische Zwecke ausgegeben. ${ }^{23}$

Im Oktober 2015 übertrug der Kreml einem anderen Amtsträger die Aufsicht über die Verwaltung des besetzten Donbass. ${ }^{24}$ Dmitri Kosak, der Vize-Ministerpräsident für regionale Angelegenheiten einschließlich eingefrorener Konflikte, übernahm die Zuständigkeit von Vladislav Surkov aus der russischen Präsidialverwaltung. Die Ernennung von Kosak, einem aus St. Petersburg stammenden Juristen, deutete auf einen Übergang von einer Kriegsverwaltung zu einem langfristigen Management hin. ${ }^{25}$ Sergej Nasarov, stellvertretender Minister für wirtschaftliche Entwicklung der Russischen Föderation, übernahm das Management der Wirtschaft der besetzten Zone. Er holte

20 Anders Åslund, „New Russian Management of the Donbas Signifies Putin May Be Ready to Negotiate”. 21 RBC Media und Jöcke in Bild-Zeitung.

22 International Crisis Group 2016.

23 „Moscow spent \$2 billion on the Terrorists in Donbas in 2016”, Liga. Novosti, 11. Mai 2017, http://news.liga.net/news/politics/14748204moskva_potratila_2_mlrd_na_terroristov_v_donbasse_v_2016_m_ godu.htm

24 Anders Åslund, „New Russian Management of the Donbas Signifies Putin May Be Ready to Negotiate”.

25 Interview mit einem ehemaligen hochrangigen Regionalbeamten des Oblast Donezk, November 2015. 
eine Vielzahl russischer Behörden mit ins Boot, während sich russische Privatunternehmen kaum engagierten. ${ }^{26}$ Am 18. Februar 2017 erließ Putin ein Dekret, wonach Russland ab sofort Reisepässe und andere von den Behörden der Volksrepubliken Donezk und Luhansk ausgestellte Dokumente anerkannte. Zuvor hatten weder Russland noch die Ukraine Abiturzeugnisse oder Universitätsexamina aus diesen Regionen anerkannt. Studenten mussten entweder nach Russland oder in die Ukraine gehen, um ihr Studium abzuschließen.

Die russische Regierung hat versucht, das Bankensystem zu erneuern. Laut einem Eintrag vom Dezember 2015 im „The Donbass Paradox“, einem investigativen Projekt von Journalismfund.eu über die Situation der Wirtschaft und der Unternehmen in den von den Rebellen gehaltenen Gebieten, sind „fast alle ausländischen Unternehmen aus der besetzten Zone geflohen. Die meisten ihrer Vermögenswerte sind inzwischen zerstört, geplündert oder besetzt worden. Alle Banken - russische, französische, österreichische, italienisch und ukrainische - haben Mitte 2014 ihre Filialen geschlossen. Die Rebellen haben viele der Niederlassungen geplündert und Geldautomaten gesprengt, um sich so Geld zu beschaffen. Die einzige Bank, die geöffnet hat, ist die von den Rebellen geführte Republikanische Zentralbank, die vor allem die ehemaligen Geschäftsräume der ukrainischen Banken PrivatBank und Ošadbank nutzt.“27 Aktiv ist auch, wie auf der Krim, die russische Staatsbank für Gebiete mit eingefrorenen Konflikten RCNB. Militärisch gesehen hat Russland die Donbass-Rebellen unter reguläres russisches Kommando gestellt.

\section{Materielle Verluste im Donbass}

Die wirtschaftliche und humanitäre Lage im Donbass ist katastrophal und steht in scharfem Gegensatz zur Situation auf der Krim. Zunächst einmal hat der Krieg im Donbass zwischen Mai und August 2014 erhebliche Zerstörungen angerichtet. Die Wirtschaftsleistung sank um ungefähr zwei Drittel. Im Anschluss an die beiden Minsker Vereinbarungen besetzten Truppen unter russischer Führung zusätzliche Hunderte von Quadratkilometern ukrainisches Territorium. Die Linie, die das von der Ukraine gehaltene Territorium und das von den Rebellen

26 Ebenda, vgl. auch Anders Åslund, „New Russian Management of the Donbas Signifies Putin May Be Ready to Negotiate".

27 Michael Bird, Lina Vdovii und Yana Tkachenko, „The Great Looting of Donbass”, EUObserver, 10. Dezember 2015, https://euobserver. com/investigations/131428. besetzte Gebiet trennt, hat sich seither etwas stabilisiert, aber an ihr entlang kommt es wieder zu Schusswechseln. Der tägliche Beschuss hat erhebliche materielle Zerstörungen angerichtet und Lieferketten unterbrochen. Die Produktion hat sich nur in geringem Umfang erholt. Bis März 2017 hatten die Rebellenregierungen in Donezk und Luhansk so gut wie alle Unternehmen, die in ihren Gebieten noch funktionierten, enteignet.

Die vierteljährlichen Berichte des UN-Hochkommissars für Menschenrechte (UNHCR) beschreiben regelmäßig ausführlich die Plünderung und Schikanierung von Besitzern von Kleinbetrieben und Immobilien, etwa der Folgende vom Sommer 2017: „Von bewaffneten Gruppen eingeführte parallele Verfahren, die Erbfälle, Verkauf und Kauf von Immobilien ,regeln', sorgen weiterhin für unnötige Härten bei der Bevölkerung [... S]ämtliche Immobiliengeschäfte, die nach dem 11. Mai 2014 vollzogen wurden, müssen bei der ,Volksrepublik Donezk' gemeldet werden.“ Und weiter: „Bewaffnete Gruppen haben das Recht zur ungestörten Nutzung von Geschäftsräumen in Privatbesitz oder anderer gewerblicher Immobilien weiter eingeschränkt. Am 5. Juli 2017 berichtete ein Mitglied des ,Volksrats‘ der ,Volksrepublik Donezk‘, 109 Privatmärkte seien seit April 2017 in ,Staatseigentum übergegangen. “28 Mehr als drei Jahre nach Beginn der Besatzung belegen diese Berichte, dass sich schwer kontrollierbare bewaffnete Gangs nach Lust und Laune fremdes Eigentum aneignen.

Die Wirtschaft im Donbass ist kollabiert. Im Sommer 2014 brach das Bruttoinlandsprodukt laut amtlichen ukrainischen Statistiken um 70 Prozent ein und es hat sich seither nicht nennenswert erholt. Kämpfer plünderten kleine Geschäfte und sämtliche Banken, die regionalen Banken stellten ihre Geschäftstätigkeit ein; viele Güter wurden zu Mangelware. Ein Bericht der International Crisis Group von 2016 beschreibt die Not und die Gesetzlosigkeit in der Region, in der ein Dutzend Gangs um die Kontrolle konkurriert. ${ }^{29}$ Im Oktober 2014 waren 2,6 Prozent des ukrainischen Staatsgebiets besetzt, auf dieses entfielen zehn Prozent des ukrainischen BIP und 15 Prozent der Industrieproduktion. ${ }^{30}$ Im März 2015 bezifferte die Weltbank die materiellen Kriegsschäden im Donbass auf insgesamt 463 Millionen Dollar, eine sehr niedrige Schätzung, wenn man das Ausmaß der Zerstörungen bedenkt. Davon entfielen 352 Millionen Dollar auf den Verkehrs- und 53 Milli-

\section{UNHCR 2017b.}

29 International Crisis Group 2016.

30 „Ukrainian Economy: Forecasts Revised to Factor in Protracted Military Conflict”, Dragon Capital, 2. Oktober 2014, www.dragoncapital.com. 


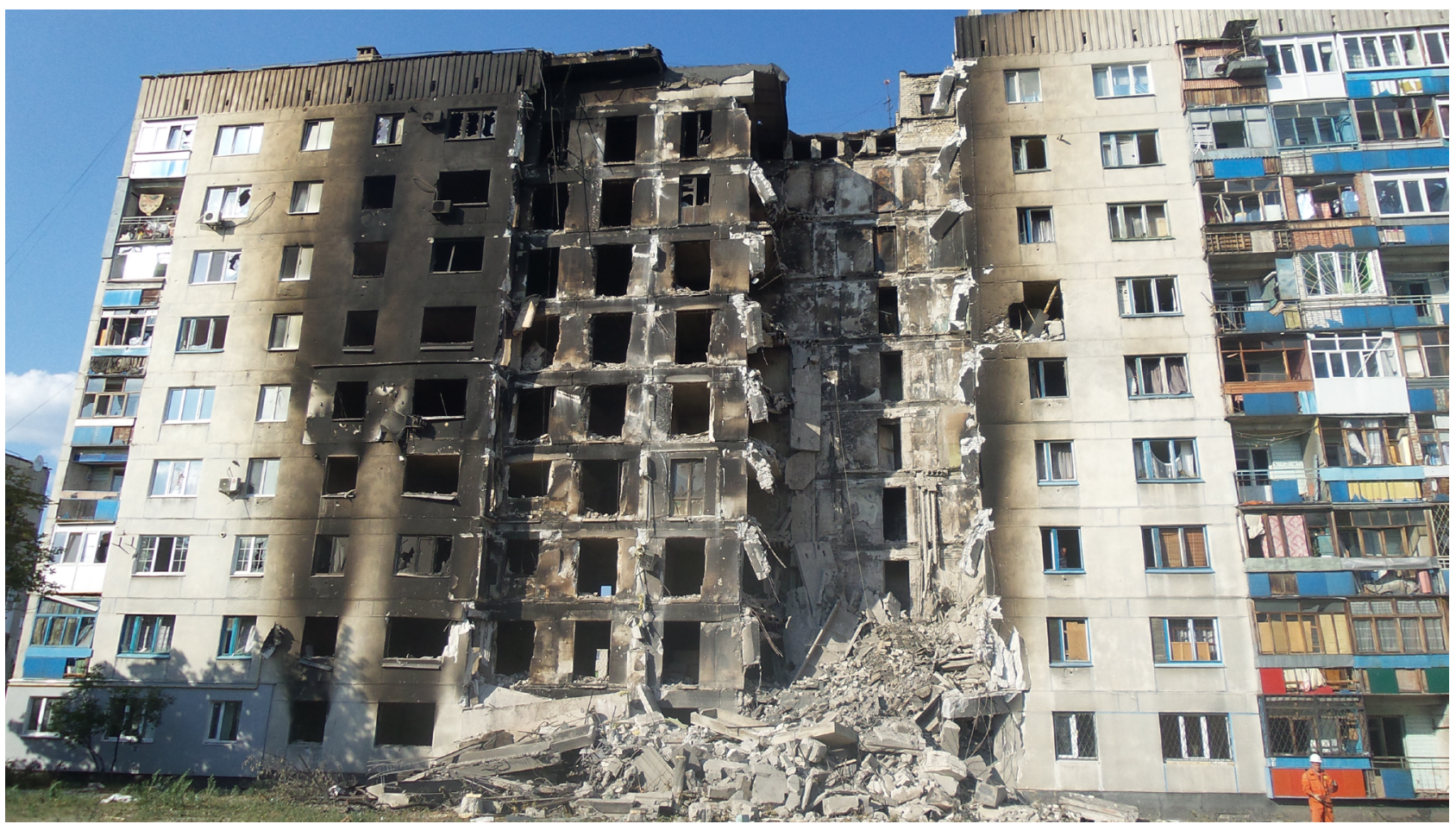

Abbildung: Zerstörungen an Häusern in der Region Lugansk, Bildnachweis: Wikimedia Commons, Lionking

onen Dollar auf den Energiesektor. ${ }^{31}$ Die Schäden an den Verkehrswegen bezogen sich auf 1.100 Kilometer öffentliche Straßen einschließlich zehn Brücken. ${ }^{32}$ Diese Zahlen werden nur selten zitiert, weil sie voreilig und offenbar auch zu niedrig waren.

Im September 2014 gab Volodymyr Groisman, der damalige Minister für Regionalentwicklung, Wohnungsbau und öffentliche Versorgung, die erste fundierte Erklärung über die Höhe der materiellen Verluste im Donbass ab. ${ }^{33}$ Er sagte, 11.325 Infrastruktureinrichtungen im Wert von insgesamt 11,9 Milliarden Hryvnja (UAH) (zur damaligen Zeit 903 Millionen Dollar) seien in den Regionen Luhansk und Donezk zerstört worden. Darunter seien 4.700 Energieanlagen, 4.500 Wohngebäude, 217 Bildungseinrichtungen, 132 Industrieanlagen und 45 medizinische Einrichtungen. Im Dezember 2014 berichtete das UNHCR, im besetzten Donbass seien etwa 12.000 öffentliche und private Anlagen und Einrichtungen zerstört worden. ${ }^{34}$ Für diese umfangreichen materiellen Zerstörungen habe es keine Entschädigung gegeben.

31 World Bank 2015.

32 Ebenda.

33 UNHCR 2014, 11.

34 „Damages Caused by the War in Ukraine”, Euromaidan Press, 21. September 2014, http://euromaidanpress.com/2014/09/21/damagescaused-by-the-war-in-east-ukraine/.
Im Donbass konzentrierten sich bedeutende ukrainische Unternehmen, die Maschinen, Bergbaugeräte, Ausrüstungsgüter für Hüttenwerke und Chemiefabriken, Verkehrsmittel, Lokomotiven und Waggons herstellen. Die folgenden großen Maschinenbau-Unternehmen wurden teilweise oder vollständig stillgelegt:

Donetskhormash PJSC und Donetsk PJSC, Horlivka Maschinenbauer PJSC, Novohorlivsk Maschinenwerke PJSC, Yasynuvata Maschinenwerke LLC, Azovmash PJSC, Stakhanov Waggonbau PJSC und das Kirov-Schmiedewerke „Tsentrokuz“ PJSC. ${ }^{35}$ Die Düngemittelfabrik Stirol stellte die Produktion ein. In den besetzten Gebieten befinden sich 115 der 150 Kohlegruben der Ukraine; die Produktion sank 2014 um 30 Prozent und in den ersten acht Monaten des Jahres 2015 um 50 Prozent im Vergleich zum Vorjahr. ${ }^{36}$

Von den vielen Stahlwerken der Ukraine waren zuvor nur die Alchevsk-Eisen- und Stahlwerke umfassend modernisiert worden. Sie gehören der Industrieunion Donbass und wurden zu Beginn des Krieges stillgelegt. Russische Truppen beschossen Brücken und Kraftwerke in den von ihnen kontrollierten Gebieten. ${ }^{37}$ Es gibt Berichte über viele weitere Fabriken, die geplündert oder demon-

35 Außenministerium der Ukraine 2015. 36 Ebenda.

37 Åslund 2015, 21. 
tiert und nach Russland geschafft wurden, aber es ist schwierig, an eindeutige Beweise zu gelangen.

Im April 2015 berichtete das Wiener Institut für Internationale Wirtschaftsvergleiche, dass sich die kriegsbedingten Schäden im Donbass auf etwa acht Prozent des ukrainischen BIP oder zehn Milliarden Dollar beliefen. ${ }^{38}$ Auf einer Konferenz in Kiew am 13. September 2015 bezifferte der damalige Ministerpräsident Arsenij Jazenjuk die Kriegsschäden an der Infrastruktur auf neun Milliarden Dollar, seither gab es viele weitere Zerstörungen. ${ }^{39}$

Innerhalb der „Volksrepubliken“ Donezk und Luhansk sind Menschen aus Ortschaften in der Nähe der Kampflinie in die beiden größten Städte Donezk und Luhansk gezogen. Die Grenze zwischen der Volksrepublik Donezk und der eigentlichen Ukraine ist relativ offen - mit vier Übergängen, während der Verkehr aus der Volksrepublik Luhansk durch eine einzige Kontrollstelle gezwängt wird..$^{40}$ Das Verkehrsaufkommen ist beträchtlich: Jeden Tag kommt es zu 25.000 Grenzübertritten, hauptsächlich von älteren Menschen, die ihre ukrainischen Renten abheben wollen. ${ }^{41}$ Einige Bewohner der besetzten Zonen überqueren die Grenze auch, um Lebensmittel einzukaufen - die Preise sind in der freien Ukraine viel niedriger und die Auswahl ist wesentlich besser. Bis März 2017 bildeten vierzig Unternehmen, die Rinat Achmetov gehörten und die rund 57.000 Menschen beschäftigten, das Rückgrat dessen, was von der Wirtschaft übrig geblieben war. Zu diesem Zeitpunkt beschloss der Kreml, diese Unternehmen auf nach wie vor unklarer Rechtsgrundlage zu enteignen. Einige lokale Geschäftsleute, die dem ehemaligen Präsidenten Viktor Yanukovič nahestehen, betreiben weiterhin Unternehmen unter dem Schutz lokaler Warlords, insbesondere Serhiy Kurčenko. ${ }^{42}$ Im Donbass illegal abgebaute Kohle ist nach Russland, in die Türkei und nach Polen exportiert worden. Im Januar 2018 belegte das US-Finanzministerium einige dieser Kohlehändler mit Sanktionen. ${ }^{43}$

\section{Adarov 2015.}

39 Aussage auf der Yalta European Strategy Conference in Kiew, September 2014.

40 UNHCR 2017c.

41 Die Zahlen über die täglichen Übertritte stammen vom ukrainischen Grenzschutz, vgl. http://dpsu.gov.ua/ua/news/1490249414situaciya-v-kontrolnih-punktah-vizdu-viizdu-na-linii-rozmezhuvannya/.

42 Pavel Kanygin, „The Donbass War. Assessing the Aftermath: How the 'Russian Spring' Came to an End in Eastern Ukraine”, Meduza, 13. November 2015.

43 US Department of the Treasury, "Treasury Sanctions Additional Individuals and Entities in Connection with the Conflict in Ukraine and Russia’s Occupation of Crimea”, 26. Januar 2018, https://home. treasury.gov/news/press-releases/sm0266.
Am 15. März 2017 beschlossen die ukrainischen Behörden, den Handel mit dem „nicht von der Regierung kontrollierten Gebiet (NGCA)“ auszusetzen, nachdem verschiedene Kriegsveteranen- und Oppositionsgruppen Bahnstrecken blockierten, die dieses mit dem Rest des Landes verbinden. Sie taten dies mit der Begründung, die Rebellen profitierten finanziell von dem Handel und dieser ziehe daher den Konflikt in die Länge. Die Blockade brachte den Transport von Kohle aus Zechen in der NGCA zum Erliegen. Stahlfabriken, andere Metallwerke und Kraftwerke in der Ukraine waren jedoch auf diese Lieferungen angewiesen. Die Rebellen übernahmen die Kontrolle über alle ukrainischen Vermögenswerte in der NGCA, darunter auch 40 mittlere und große Unternehmen. Daraufhin untersagte Kiew sämtliche kommerziellen Transporte von Gütern zwischen der NGCA und dem Rest der Ukraine. Die Nationalbank der Ukraine schätzte, dass dies $\mathrm{zu}$ einem Rückgang des nationalen BIP um 1,3 Prozent führen würde - 0,7 Prozent des BIP, weil die enteigneten Unternehmen nicht länger Teil der ukrainischen Volkswirtschaft sind, und 0,6 Prozent des BIP wegen der Aussetzung des Handels. ${ }^{44}$ Im November 2017 berichtete das UNHCR: „Eines der dringlichsten, nach wie vor ungelösten sozioökonomischen Probleme ist die Tatsache, dass zerstörtes oder beschädigtes Eigentum nicht rückerstattet und instandgesetzt wird bzw. keine Entschädigung dafür gezahlt wird.“ Die Kommission stellte fest, es habe „keine Fortschritte bei der Erstellung eines einheitlichen Registers beschädigter und/oder zerstörter Immobilien gegeben“. ${ }^{45}$

Bevor sie aus dem Amt gedrängt wurde, hatte die Regierung Yanukovič Production-Sharing Agreements (PSA) mit Chevron in der Westukraine und Royald Dutch Shell im Donbass über die Erkundung und Förderung von Schiefergas abgeschlossen. Bei seinem Rückzug daraus berief sich Shell angesichts seiner in der Kriegszone gelegenen Felder auf höhere Gewalt. ${ }^{46}$ (Es sollte hinzugefügt werden, dass noch andere Faktoren zu dieser Erklärung beitrugen, insbesondere der Einbruch des Ölpreises im Sommer 2014 und die schlechten Rahmenbedingungen für ausländische Energieinvestitionen in der Ukraine.) Zuvor hatte Shell davon gesprochen, fünf bis zehn Milliarden Dollar in dieses Feld zu investieren. ${ }^{47}$

44 David Saha, „The effect of company seizures and trade suspension in Donbas", German Advisory Group Ukraine Newsletter, August 2017, https://www.beratergruppe-ukraine.de/wordpress/wp-content/ uploads/2017/08/Newsletter_106_2017_German-Advisory-Group.pdf. 45 UNHCR 2017c.

46 Åslund 2015, 202.

47 Gespräche mit dem für die Ukraine verantwortlichen Shell-Manager im September 2014. 


\section{Russische Handelssanktionen und ihre Kosten}

Der wirtschaftliche Indikator, in dem sich der Schaden für die ukrainische Wirtschaft infolge der russischen Aggression am deutlichsten widerspiegelt, ist der Außenhandel. Es gab viele Rückschläge. Zunächst einmal verhängte Russland im Juli und August 2013 mehrere Handelssanktionen gegen die Ukraine, insbesondere gegen Einfuhren landwirtschaftlicher Erzeugnisse und von Stahl. Anfangs richteten sich diese Sanktionen gegen proeuropäische ukrainische Geschäftsleute, aber im Lauf des Jahres 2014 wurden sie stetig ausgeweitet und betrafen schließlich die gesamte Ukraine.

Russlands bevorzugtes geopolitisches Werkzeug ist Gazprom, man spielt sowohl mit den Preisen als auch mit den Liefermengen, um damit politische Ziele zu erreichen. Im ersten Quartal 2014 berechnete Gazprom der Ukraine entsprechend einer Vereinbarung, die die Präsidenten Putin und Yanukovič am 17. Dezember 2013 geschlossen hatten, 268,50 Dollar je 1.000 Kubikmeter. Am 1. April erhöhte Gazprom den Preis auf 385 Dollar je 1.000 Kubikmeter. Der Konzern erklärte, er werde keinen speziellen Yanukovič-Rabatt mehr gewähren. Zwei Tage später hob Gazprom den Preis erneut an, um weitere 100 Dollar auf 485 Dollar je 1000 Kubikmeter. Das Unternehmen behauptete, da Russland die Krim annektiert habe, sei es nicht länger verpflichtet, einen Rabatt für den von Russland gepachteten Flottenstützpunkt Sewastopol anzubieten, wie es Putin im April 2010 in Charkiv mit Yanukovič vereinbart hatte. ${ }^{48}$ Daraufhin stellte Kiew die Zahlungen ein und am 16. Juni 2014 stoppte Gazprom die Gaslieferungen an die Ukraine. ${ }^{49}$

Am selben Tag reichten beide Unternehmen beim Schiedsgerichtsinstitut der Stockholmer Handelskammer, dem vereinbarten Gerichtsstand für etwaige geschäftliche Streitigkeiten zwischen ihnen, Schadensersatzklagen über mehrere Milliarden Dollar gegeneinander ein. Im November 2015 stellte Naftogaz die Gaseinfuhr von Gazprom ein. Im Mai 2017 gewann Naftogaz seinen Stockholmer Rechtsstreit gegen Gazprom; der russische Gigant ging in Revision, die im Dezember 2017 zu einer Entscheidung führte, die beide Seiten als Sieg hinstellten, tatsächlich aber wurde das Urteil zugunsten von Naftogaz bestätigt. ${ }^{50}$

48 Åslund, 2015, 205.

49 Vladimir Socor, „Russia Suspends Natural Gas Supplies to Ukraine”, Eurasia Daily Monitor, 19. Juni 2014, https://jamestown.org/ program/russia-suspends-natural-gas-supplies-to-ukraine/.

50 „Naftogaz wins gas sales arbitration case against Gazprom on all issues in dispute", UNIAN, 22. Dezember 2017, https://econo-
Nach der russischen Annexion der Krim im März 2014 verlor die Ukraine einen Großteil ihrer Exportkapazität, und im Sommer war die Wirtschaft im Donbass aufgrund des Krieges praktisch lahmgelegt. Die erhebliche Produktion von Stahl, Kohle, Eisenerz und damit verbundenen Produkten kam im Donbass fast völlig zum Erliegen, ebenso die Ausfuhr von Stahl und anderen Produkten aus der Region. Auch die Verkehrswege waren unterbrochen. Im September 2014 reagierte die Ukraine mit umfangreichen Sanktionen. So verringerte sie insbesondere ihre Rüstungsexporte nach Russland. Im Januar 2016 trat das Abkommen über eine Vertiefte und umfassende Freihandelszone (Deep and Comprehensive Free Trade Area, DCFTA) zwischen der EU und der Ukraine in Kraft. Daraufhin setzte Russland das bestehende multilaterale Freihandelsabkommen zwischen der Gemeinschaft unabhängiger Staaten in Bezug auf die Ukraine außer Kraft, das heißt es verhängte Einfuhrzölle gegen die Ukraine. ${ }^{51}$ Obendrein führte Russland Transitbeschränkungen für ukrainische Exporte nach Kasachstan und Kirgisistan ein, die den Gesamtwert der ukrainischen Exporte um 0,4 Prozent reduzierten. ${ }^{52}$ Die beiden Länder haben ihre vielen Handelssanktionen schrittweise gesteigert.

Tabelle: Exporte und Importe der Ukraine in 2012 und 2016, in Milliarden US-Dollar

\begin{tabular}{llll}
\hline & $\mathbf{2 0 1 2}$ & $\mathbf{2 0 1 6}$ & Rückgang \\
\hline Gesamtausfuhr & 68,7 & 36,3 & 47 Prozent \\
nach Russland & 17,6 & 3,6 & 80 Prozent \\
Gesamteinfuhr & 84,7 & 39,2 & 54 Prozent \\
aus Russland & 27,4 & 5,1 & 81 Prozent \\
\hline
\end{tabular}

Quelle: IMF Direction of Trade Statistics

Vorstehende Tabelle zeigt einen schockierenden Einbruch des gesamten Außenhandels der Ukraine. Das letzte normale Jahr war 2012, vor den Handelssanktionen und der militärischen Aggression Russlands. Dessen Anteil am Außenhandel der Ukraine fiel von 29 Prozent in 2012 auf 11,5 Prozent in 2016. Diese Handelsrückgänge haben der ukrainischen Wirtschaft schwer geschadet. 2016 markierte den Tiefpunkt; im Jahr 2017 legte der ukrainische Außenhandel deutlich zu, obwohl Russland seine Sanktionen nicht lockerte.

mics.unian.info/2313624-naftogaz-wins-gas-sales-arbitration-caseagainst-gazprom-on-all-issues-in-dispute.html.

51 „Russia suspends free-trade deal with Ukraine from 2016”, Agence France-Presse, 16. Dezember 2015, https://www.yahoo.com/news/ russia-suspends-free-trade-deal-ukraine-2016-154025521.html. 52 Movchan/Saha/Kirchner 2018. 
Innerhalb der ehemaligen sowjetischen Einflusssphäre hat Russland immer wieder ohne oder mit nur kurzfristiger Vorankündigung einschneidende Handelssanktionen verhängt. Anfang der 1990er-Jahre trafen diese die mittelund osteuropäischen Länder, insbesondere die baltischen Staaten. Sie alle haben daraufhin ihren Außenhandel von Russland auf die EU verlagert. ${ }^{53} \mathrm{Im}$ Jahr 2006 verhängte Russland harte Handelssanktionen gegen Moldawien und Georgien. Der Anteil der moldawischen Exporte, der nach Russland ging, sank von 40 Prozent im Jahr 2005 auf 16 Prozent in 2007, während die Gesamtausfuhr wuchs und die moldawischen Exporte in die EU schnell anstiegen. Georgien erlebte einen ähnlichen, wenn auch weniger jähen Rückgang im Handel mit Russland, von 18 Prozent der Exporte auf vier Prozent, während seine gesamte Ausfuhr um 42 Prozent in die Höhe schnellte. ${ }^{54}$

In dem Maße, wie sich der Schwerpunkt der ukrainischen Exporte von Russland zur EU verlagerte, änderte sich auch die Struktur dieser Ausfuhren, wie es bereits zuvor in Mittel- und Osteuropa der Fall gewesen war. Die ukrainischen Ausfuhren werden von vier Kategorien von Gütern dominiert: Stahl, Maschinen, landwirtschaftliche Erzeugnisse und Chemikalien. Der Anteil von Stahl an den Exporten wird wahrscheinlich weiter fallen, Chemikalien werden vielleicht ganz von der Liste verschwinden, da ihre Produktion auf billiges Gas aus Russland angewiesen ist, das wohl endgültig versiegt ist. Dagegen dürfte die Ukraine mehr landwirtschaftliche Erzeugnisse exportieren und es besteht die Hoffnung, dass sie in die europäische Lieferkette von Industriegütern und Dienstleistungen integriert wird. ${ }^{55}$

\section{Von der Ukraine eingereichte Klagen}

Die Ukraine und Russland liefern sich auch erbitterte juristische Auseinandersetzungen: So sind Klagen vor dem Stockholmer Schiedsgerichtsinstitut, dem Ständigen Schiedshof in Den Haag, dem High Court in London, dem Europäischen Gerichtshof für Menschenrechte in Straßburg, dem Internationalen Gerichtshof (IGH) und dem Internationalen Strafgerichtshof (ICC) anhängig.

53 Åslund/Warner 2004.

54 Åslund 2015, 48.

55 Anders Åslund, „What Will Happen to Russian-Ukrainian Trade after the War in Donbass", Peterson Institute for International Economics, 3. Oktober 2014. https://piie.com/commentary/op-eds/whatwill-happen-russian-ukrainian-trade-after-war-donbass.
Die wichtigsten Fälle waren die Klagen von Naftogaz und Gazprom gegeneinander vor dem Stockholmer Schiedsgerichtsinstitut. Am 31. Mai 2017 entschied es in der Hauptsache, die sich auf Zahlungen und Lieferverpflichtungen aus einem 2009 unterzeichneten, zehnjährigen bilateralen Gasabkommen bezog, zugunsten von Naftogaz. Der Wert der potenziellen Forderungen wird unterschiedlich veranschlagt, aber Naftogaz behauptete, es habe sich russischen Forderungen in Höhe von 75 Milliarden Dollar entzogen. ${ }^{56}$

Das Londoner Gericht entschied für Russland, das die Rückzahlung einer von der Ukraine nicht mehr bedienten, im Dezember 2013 - gegen Ende der Ära Yanukovič - ausgegebenen Anleihe über drei Milliarden Dollar forderte. ${ }^{57}$ Im Anschluss an die Aggressionen auf der Krim und im Donbass lehnte Russland im Unterschied zu anderen internationalen Gläubigern eine Umschuldung ukrainischer Staatsschulden ab. Kiew behauptete, die Entscheidung Russlands sei politisch motiviert, aber das Gericht wies dieses Argument in seinem Urteil vom März 2017 zurück. Zum gegenwärtigen Zeitpunkt ist die von der Ukraine eingereichte Revision noch anhängig.

Im Januar 2016 verhängte die Anti-Monopol-Kommission der Ukraine gegen Gazprom wegen Missbrauchs seiner Monopolstellung auf dem Transitmarkt eine Geldstrafe in Höhe von drei Milliarden Dollar. Anschließende Gerichtsentscheidungen erhöhten diese Geldstrafe auf sechs Milliarden Dollar, die Kiew durch Einziehung und Liquidation der Vermögenswerte von Gazprom in der Ukraine eintreiben will. ${ }^{58}$ Eine Reihe von Klagen mit Bezug zur Krim wurden von ukrainischen Unternehmen eingereicht, die dabei oftmals in Gruppen agierten. Die Rechtsgrundlage dieser Klagen ist das Bilaterale Investitionsabkommen (BIT) zwischen der Ukraine und Russland vom 27. November 1998. Sein Artikel 5 regelt Enteignungen:

1. Die Investitionen von Investoren beider vertragschließenden Parteien, die auf dem Territorium der jeweils anderen Vertragspartei getätigt werden, sollen nicht Enteignung, Verstaatlichung oder anderen Maßnah-

56 Pavel Polityuk, „Ukraine and Russia both claim victory in gas dispute”, Reuters, 22. Dezember 2017, https://www.reuters.com/article/ us-ukraine-crisis-russia-gazprom/ukraine-and-russia-both-claimvictory-in-gas-dispute-idUSKBN1EG1IO.

57 Jeremy Hodges, „Russia Wins Early Verdict in \$3 Billion Ukrainian Bond Case”, Bloomberg, 29. März 2017, https://www.bloomberg. com/news/articles/2017-03-29/russia-wins-early-verdict-in-case-over3-billion-ukrainian-bond.

58 „Ukraine to hire foreign advisers to recover US\$6 bln from Russia’s Gazprom in anti-trust case”, Unian, 23. Januar 2017, https://www. magglr.com/news/ukraine-to-hire-foreign-advisers-to-recover-us6bln-from-russias-gazprom-in-anti-trust-case/. 
men, die in ihren Folgen einer Enteignung gleichkommen (im Folgenden „Enteignung“ genannt), unterworfen werden - mit Ausnahme der Fälle, in denen solche Maßnahmen nicht diskriminierend sind und eine unverzügliche, angemessene und effektive Entschädigung erfolgt.

2. Die Entschädigung soll dem jeweils aktuellen Marktwert der enteigneten Investitionen unmittelbar vor dem Zeitpunkt der Enteignung oder zu dem Zeitpunkt, $\mathrm{zu}$ dem die Enteignung offiziell bekanntgemacht worden ist, entsprechen. Die Entschädigung soll unverzüglich, unter gebührender Berücksichtigung der Zinsen, ausgezahlt werden, die vom Zeitpunkt der Enteignung bis zum Zeitpunkt der Zahlung in Rechnung gestellt werden sollen, und zwar zu dem am Londoner Interbankenmarkt aktuell gültigen Zinssatz für dreimonatige Einlagen in US-Dollar (LIBOR) plus ein Prozent, und sie soll effizient verwertbar und frei übertragbar sein. ${ }^{59}$

Artikel 9 des BIT räumt die Möglichkeit ein, verschiedene Schiedsgerichte anzurufen. Dabei kann es sich um „ein zuständiges Gericht oder ein Schiedsgericht jener Vertragspartei [handeln], auf deren Hoheitsgebiet die Investitionen getätigt wurden“, „das Schiedsgerichtsinstitut der Stockholmer Handelskammer“ oder „ein ,Ad hoc“Schiedsgericht, das entsprechend der Schiedsgerichtsordnung der UN-Kommission für internationales Handelsrecht (UNCITRAL) entscheidet“. ${ }^{60}$ Bislang scheinen sich alle ukrainischen Unternehmen an den Ständigen Schiedshof in Den Haag gewandt zu haben.

Die Zahl der ukrainischen Klagen ist hoch und wird wahrscheinlich zunehmen, wenn die Kläger erfolgreich sind. Der größte Prozess wurde von Naftogaz und sechs seiner Tochtergesellschaften (Chernomornaftogaz, Ukrtransgaz, Likvo, Ukrgazdobuvanya, Ukrtransnafta und Gaz of Ukraine) angestrengt. Die Unternehmen fordern fünf Milliarden Dollar als Ersatz für Schäden, die ihnen durch Beschlagnahme ihrer Vermögenswerte auf der Krim entstanden sind, zuzüglich zwei Milliarden Dollar Zinsen. Die Russische Föderation hat die Zuständigkeit des Internationalen Schiedsgerichtshofs in Paris nicht anerkannt, aber im Juli 2017 hat der Ständige

59 „Agreement Between the Government of the Russian Federation and the Cabinet of Ministers of the Ukraine on the Encouragement and Mutual Protection of Investments”,(Moskau, 27. November 1998), Quelle: Investment Policy Hub, http://investmentpolicyhub.unctad. org/Download/TreatyFile/2233.

60 Ebenda.
Schiedshof (PCA) in Den Haag entschieden, dass er die Klagen annimmt. ${ }^{61}$

Mindestens sieben weitere Zivilprozesse wurden wegen der Beschlagnahme von Vermögenswerten auf der besetzten Krim gegen die Russische Föderation angestrengt und zwar von Unternehmen wie Ukrnafta, Ošadbank und Privatbank. Die staatseigene Ošadbank fordert eine Milliarde Dollar Schadensersatz. ${ }^{62}$ Privatbank wird wahrscheinlich einen ähnlich hohen Betrag fordern, ebenso Ukrnafta. DTEK, der größte private Strom- und Kohleproduzent der Ukraine, hat erklärt, er werde von Russland 500 Millionen Dollar Schadensersatz für den Verlust seiner Vermögenswerte auf der Krim verlangen. ${ }^{63}$ Die anhängigen Forderungen ukrainischer Unternehmen könnten Schadensersatzzahlungen von über zehn Milliarden Dollar einbringen.

Die Forderungen ukrainischer Unternehmen auf der Krim scheinen auf stichhaltigen rechtlichen Anspruchsgrundlagen $\mathrm{zu}$ basieren. Russland hat die Krim offiziell annektiert, das BIT galt unbestreitbar zum damaligen Zeitpunkt. Die Unternehmen haben einen wünschenswerten Gerichtsstand gefunden; Moskau weigert sich, die Zuständigkeit des Ständigen Schiedshofs in Den Haag anzuerkennen, aber es ist nicht in der Lage, seine ablehnende Haltung durch glaubwürdige Argumente zu stützen. Wenn ein ukrainisches Unternehmen vor einem internationalen Schiedsgericht gewinnt, kann es in einem anderen Land als Russland, in dem russische Vermögenswerte vorhanden sind, Forderungen gegen die Russische Föderation erheben und zwar auf Grundlage des New Yorker Übereinkommens über die Anerkennung und Vollstreckung ausländischer Schiedssprüche von $1958 .^{64}$

Im Donbass dagegen sind die rechtlichen Verhältnisse viel unsicherer. Obgleich es offensichtlich ist, dass die russische Regierung das Gebiet kontrolliert, gibt sie dies nicht zu. Da sich Moskau weigert, offiziell die Verantwor-

61 „Demands of Ukraine’s Naftogaz against Russia Reach \$7 Billion”, Russian Legal Information Agency, 21. September 2017, http:// www.rapsinews.com/judicial_news/20170921/280188520.html.

62 Jack Newsham, „Ukrainian Bank Says \$1B Crimea Arbitration Fast-Tracked”, Law 360, 26. August 2016, https://www.law360.com/ articles/833151/ukrainian-bank-says-1b-crimea-arbitration-fast-tracked.

63 „Ukraine’s DTEK to Seek $\$ 500 \mathrm{mln}$ from Russia for Crimea Losses”, Reuters, 11. April 2017, https://uk.reuters.com/article/ukrainedtekcrimea-russia/ukraines-dtek-to-seek-500-mln-from-russia-forcrimea-losses-idUKL8N1HJ33G.

64 Sergejs Dilevka und Mena Chambers, „Arbitration Claims by Ukrainian Investors under the Russian-Ukraine BIT", CIS Arbitration Forum, 17. Februar 2016, http://www.cisarbitration.com/2016/02/17/ arbitration-claims-by-ukrainian-investors-under-the-russia-ukrainebitbetween-crimea-and-a-hard-place/. 
tung für die besetzten Gebiete zu übernehmen, ist unklar, ob das ukrainisch-russische BIT anwendbar ist. Und es ist auch nicht klar, in welchem Land Forderungen gerichtlich geltend gemacht werden könnten. Eine russisch-ukrainische Vereinbarung über den Donbass müsste eine Klarstellung der rechtlichen Zuständigkeit enthalten.

System Capital Management (SCM), eine in Donezk ansässige Industrie- und Finanzbeteiligungsgesellschaft, die die höchsten Forderungen in Bezug auf entzogene Vermögenswerte im Donbass erhebt, hat begonnen, in Drittländern wie der Türkei gegen Unternehmen zu prozessieren, die von Dieben aus SCM-Zechen in den besetzten Gebieten gestohlene Kohle gekauft haben. ${ }^{65}$

Regierungen haben andere Möglichkeiten, um sich gegenseitig $\mathrm{zu}$ verklagen. Die bekannteste Institution für solche Fälle ist der Internationale Gerichtshof in Den Haag. Es ist die höchste gerichtliche Instanz für Streitigkeiten, bei denen nationale Hoheitsrechte von Staaten betroffen sind. Die Ukraine reichte beim IGH Klage gegen Russland wegen Verstoßes gegen UN-Konventionen ein. ${ }^{66}$

Im Hinblick auf den Donbass beschuldigt die Regierung in Kiew Russland, gegen das Übereinkommen zur Bekämpfung der Finanzierung des Terrorismus verstoßen $\mathrm{zu}$ haben. Sie bezichtigt es der Beteiligung am Abschuss von Malaysia Airlines Flug Nr. 17 über dem besetzten Gebiet am 17. Juli 2014. ${ }^{67}$ Die Ukraine fordert, dass der IGH die Russische Föderation anweist, „sofort und bedingungslos jegliche Unterstützung einschließlich der Bereitstellung von Geldern, Waffen und Ausbildung für illegale bewaffnete Gruppen, die Terrorakte in der Ukraine verüben, einschließlich Kräften der Volksrepubliken Donezk und Luhansk, einzustellen und zu unterlassen." ${ }^{68}$

Sich auf das Übereinkommen zur Bekämpfung der Finanzierung des Terrorismus berufend, hat die Ukraine von Russland auch umfassende Reparationen verlangt für den Abschuss von Flug Nr. 17, den Beschuss von Zivilisten in Volnovacha, Mariupol und Kramatorsk, die Bombardierung von Zivilisten in Charkiv und ,alle anderen Ter-

65 Persönliche Mitteilung von Jock Mendoza-Wilson von SCM, Februar 2018.

66 Gregorio Baggiani, „Ukraine’s Legal Cases Against Russia in International Courts”, Eurasia Daily Monitor, 1. März 2017, https:// jamestown.org/program/ukraines-legal-cases-russia-internationalcourts/.

67 Roman Goncharenko, „Ukraine v. Russia: A Potential GameChanging Lawsuit Comes before the ICJ”, Deutsche Welle, 5. März 2017, http://www.dw.com/en/ukraine-v-russia-a-potential-gamechanging-lawsuit-comes-before-the-icj/a-37806132.

68 Internationaler Gerichtshof (ICJ), „Ukraine institutes proceedings against the Russian Federation and requests the Court to indicate provisional measures”, Pressemitteilung, 17. Januar 2017, http://www.icjcij.org/files/case-related/166/19310.pdf. rorakte, die die Russische Föderation durch ihre Finanzierung des Terrorismus verursacht, begünstigt oder unterstützt hat, und dafür, dass sie es unterlassen hat, die Finanzierung des Terrorismus zu unterbinden und Ermittlungen darüber anzustellen.“69

Die ukrainische Regierung stützte ihre beiden Hauptvorwürfe gegen Russland in Bezug auf die Krim auf das Internationale Übereinkommen zur Beseitigung jeder Form von Rassendiskriminierung (in erster Linie in Bezug auf Krimtartaren, aber auch ethnische Ukrainer auf der Krim) und das Seerechtsübereinkommen der Vereinten Nationen, „betreffend die Ausbeutung ukrainischer Fischbestände durch Russland, die Behinderung der freien Schifffahrt ukrainischer Seefahrzeuge in den Gewässern der Krim und in deren Nähe sowie den Bau von Infrastruktureinrichtungen durch Russland, dem die Ukraine nicht zugestimmt hat" ${ }^{70}$

Russland hat erwidert, dass es sich keine Verstöße gegen das Völkerrecht zuschulden kommen ließ, und weigert sich daher, sein Vorgehen im Donbass zu „legitimieren", indem es sich vor dem Internationalen Gerichtshof verteidigt. ${ }^{71}$ Es hat seine formale Antwort auf kurze Stellungnahmen beschränkt wie zum Beispiel: „Die Russische Föderation fordert den Gerichtshof auf, den von der Ukraine vorgelegten Antrag auf Erlass einstweiliger Maßnahmen abzulehnen."72

Ein alternatives internationales Gericht ist der Europäische Gerichtshof für Menschenrechte (EGMR), der mit dem Europarat verbunden ist. Das ukrainische Justizministerium hat wiederholt erklärt, es werde sich an den EGMR wenden, um von Russland Schadensersatz für die wirtschaftlichen Verluste der Ukraine auf der Krim zu verlangen. Im Dezember 2015 verabschiedete die russische Staatsduma jedoch ein Gesetz, das die russische Regierung befugt, Urteile des EGMR für ungültig zu erklären, sodass diese wahrscheinlich kein Urteil des europäischen Gerichts anerkennen wird. ${ }^{73}$ Dennoch erlaubt das New Yorker Übereinkommen erfolgreichen Klägern, russisches Eigentum außerhalb Russlands zu pfänden. Über den

69 Ebenda.

70 Gregorio Baggiani, „Ukraine’s Legal Cases Against Russia in International Courts”, Eurasia Daily Monitor, 1. März 2017, https:// jamestown.org/program/ukraines-legal-cases-russia-internationalcourts/.

71 Ebenda.

72 Application of the International Convention for the Suppression of the Financing of Terrorism and of the International Convention on the Elimination of All Forms of Racial Discrimination (Ukraine v. Russian Federation), ICJ-Pressemitteilung, 9. März 2017, http://www. icjcij.org/files/case-related/166/19372.pdf.

73 Baggiani, „Ukraines’ Legal Cases”. 
EGMR hat sich eine regelrechte Flut von ukrainischen Klagen ergossen. Im Januar waren bei der Registratur des Gerichts rund 3.800 Klageschriften hinterlegt, von denen die meisten die russische Aggression betrafen; hinzu kommen fünf zwischenstaatliche Klagen, die die Ukraine gegen Russland eingereicht hat. ${ }^{74}$

Der Abschuss des Flugzeugs von Malaysia Airlines ist ein Sonderfall. Die Niederlande haben sich für zuständig erklärt, weil das Flugzeug von Amsterdam aus startete und die meisten seiner Passagiere niederländische Staatsbürger waren. Allerdings kamen die niederländischen Ermittlungen nur sehr langsam voran und ukrainische Behörden haben erwogen, die Sache vor die Internationale Handelskammer (ICC) zu bringen. ${ }^{75}$ Vorsorglich zog sich Russland im November 2016 aus der ICC zurück. ${ }^{76}$

Die unklare Situation im Donbass hat die juristische Aufarbeitung erschwert. Auf der Krim übernahm Russland durch seine Annexion die juristische Verantwortung; in Donezk und Luhansk hat es dies nicht getan. Niemand erkennt die „Volksrepubliken“ Donezk und Luhansk an, sie sind nicht Mitglied einer internationalen Rechtsinstitution, was es schwer macht, sie gerichtlich zur Verantwortung zu ziehen. In gleicher Weise erfolgten Beschlagnahmen auf der Krim nach russischem Gesetz, während Beschlagnahmen durch Kräfte der beiden „Volksrepubliken“ weniger förmlicher Natur sind.

Am 18. Januar 2018 verabschiedete das ukrainische Parlament ein neues Gesetz ,über die Wiedereingliederung des Donbass“. Es änderte dessen Rechtsstellung grundlegend. Bis dahin hatten die ukrainischen Behörden die militärischen Aktivitäten im Donbass „Terrorismus“ genannt und daher Russland beschuldigt, Terrorismus zu finanzieren. Jetzt nennt Kiew Russland einen Aggressor und die nicht von der Regierung kontrollierten Gebiete „vorübergehend besetzt“. Dies ändert den völkerrechtlichen Bezugsrahmen und eröffnet ukrainischen Klägern neue Optionen.

74 ,ECHR is considering 3,800 complaints by the Ukraine due to the consequences of Russian aggression", Evropeiska Pra$v d a$, 25. Januar 2018, https://www.eurointegration.com.ua/rus/ news/2018/01/25/7076603/.

75 Aaron Matta, „MH17 Downing Suspects to be Prosecuted Before Dutch Domestic Courts - An Obstacle or an Advantage for International Justice?”, Opinio Juris, 17. Juli 2017, http://opiniojuris. org/2017/07/17/33212/.

76 Baggiani, „Ukraine’s legal Cases”.

\section{Schlussfolgerungen}

Es ist schwer, eine gesicherte Zahl vorzulegen, die die Gesamtverluste der Ukraine infolge der russischen Aggressionen seit 2014 genau beziffert. Man kann aber zumindest einen groben Betrag angeben, indem man eine von dem Wirtschaftswissenschaftler Thomas Piketty benutzte Methode anwendet. Dieser hat in seinem Buch „Das Kapital im 21. Jahrhundert“ geschätzt, dass der Gesamtwert des Vermögens in Europa im Laufe der vergangenen 140 Jahre im Durchschnitt etwas mehr als dem Vierfachen des Bruttoinlandsprodukts (BIP) entsprach. ${ }^{77}$ Das einfachste und umfassende Maß für die Verluste, die die Ukraine erlitten hat, erhält man daher, wenn man den Prozentsatz des BIP, den die Ukraine eingebüßt hat, mit vier multipliziert. Der Donbass steuerte zehn Prozent zum BIP der Ukraine bei und die Krim 3,7 Prozent. ${ }^{78}$ Der Internationale Währungsfonds (IWF) bezifferte das BIP der Ukraine im Vorkrisenjahr 2013 auf 179,6 Milliarden Dollar. ${ }^{79}$ Daher würde sich der Gesamtwert des ukrainischen Vermögens auf der Krim und im Donbass auf 13,7 Prozent von 179,6 Milliarden Dollar, multipliziert mit vier, also 98,4 Milliarden Dollar belaufen. Der Vermögensverlust im Donbass betrüge 71,8 Milliarden Dollar, der Verlust auf der Krim 26,6 Milliarden. Dies entspricht ungefähr dem Vermögensverlust, den die Ukraine erleidet, wenn sie diese Gebiete dauerhaft verliert.

Der ukrainische Staat, ukrainische Unternehmen und ukrainische Privatpersonen haben aufgrund der russischen Aggression erhebliche Vermögensverluste auf der Krim und im Donbass erlitten. Schon jetzt sind viele Prozesse anhängig, ihre Zahl wird in den kommenden Jahren mit Sicherheit noch beträchtlich steigen. Dies wird die Außen- und Wirtschaftspolitik der Ukraine maßgeblich prägen. Aus diesem Grund muss diesen Fragen weitreichende politische Beachtung geschenkt werden.

Insbesondere sollten die USA und die EU mit der Ukraine und Russland darauf hinarbeiten, eine Übereinkunft über den Donbass zu erreichen, in der Moskau die volle finanzielle Verantwortung für die Zerstörungen übernimmt. Außerdem sollte der Westen sicherstellen, dass die Frage russischer Reparationen Teil einer Vereinbarung über die Rückgabe der Krim und des Donbass an die Ukraine ist. Des Weiteren sollten die USA und die EU die Sanktionen gegen Russland wegen seiner Annexion der

\section{Piketty 2013, 165.}

78 „Ukrainian Economy: Forecasts Revised to Factor in Protracted Military Conflict”, Dragon Capital, 2. Oktober 2014, www.dragoncapital.com.

79 IMF 2017. 
Krim und der militärischen Aggression im Donbass aufrechterhalten, bis es diese Gebiete an die Ukraine zurückgegeben und ukrainische Unternehmen für die erlittenen materiellen Verluste angemessen entschädigt hat.

\section{Literatur}

Adarov, Amit et al. (2015): How to Stabilise the Economy of Ukraine, Wien: Vienna Institute for International Economic Studies, https://wiiw.ac.at/how-to-stabilise-the-economy-ofukraine-dlp-3562.pdf.

Åslund, Anders (2015): Ukraine: What Went Wrong and How to Fix It, Washington, DC: Peterson Institute for International Economics.

Åslund, Anders/Warner, Andrew (2004): „The EU Enlargement: Consequences for the CIS Countries“, in: Marek Dabrowski/Ben Slay/Jaroslaw Neneman (Hrsg.), Beyond Transition: Development Perspectives and Dilemmas, Aldershot: Ashgate, 231-252.

Bentzen, Naja (2015): Ukraine's economic challenges: From ailing to failing? Brüssel: European Parliamentary Research Service, http://www.europarl.europa.eu/RegData/etudes/ IDAN/2015/559497/EPRS_IDA(2015)559497_EN.pdf.

BP - British Petroleum (2014): Statistical Review for Energy. London: BP, https://www.bp.com/content/dam/bp-country/de_de/ PDFs/brochures/BP-statisticalreview-of-world-energy2014-full-report.pdf.

Bugriy, Maksym (2014): „The Cost to Ukraine of Crimea’s Annexation“, Eurasia Daily Monitor, 14. April, https://jamestown.org/ program/the-cost-to-ukraine-of-crimeas-annexation/

International Crisis Group (2016): Russia and the Separatists in Eastern Ukraine, Brüssel, https://www.crisisgroup.org/ europecentral-asia/eastern-europe/ukraine/russia-andseparatists-eastern-ukraine.

International Monetary Fund (2017): World Economic Outlook Database. Washington, D.C. IMF, https://www.imf.org/ external/pubs/ft/weo/2017/02/

Ministerium für Auswärtige Angelegenheit der Ukraine (2015): Kremlin's Black Book: Russian War Against Ukraine, Kiew: MFA, http://mfa.gov.ua/mediafiles/sites/cuba/files/Kremlin_Black_ Book_English_Oct_2015_ENG.pdf.
Movchan,Veronika/Saha, David/Kirchner, Robert (2018): The Impact of Russia's Transit Restrictions on Ukraine's Exports to Kazakhstan and Kyrgyzstan, Berlin: German Advisory Group, Policy Briefing Series.

Piketty, Thomas (2013): Capital in the Twenty-First Century, Cambridge, MA: Belknap. (dt. Das Kapital im 21. Jahrhundert, München: C.H.Beck 2014).

Saha, David/Kravchuk, Vitaliy/Movchan, Veronika (2018): The Impact of the New Kerch Strait Bridge on Ukraine's Trade, Berlin: German Advisory Group, Policy Briefing Series, https:// www.beratergruppe-ukraine.de/wordpress/wp-content/ uploads/2018/03/PB_02_2018_en.pdf.

UNHCR - Office of the United Nations High Commissioner for Human Rights (2014): Report on the human rights situation in Ukraine, 15 December 2016, New York: United Nations, https://www. ohchr.org/Documents/Countries/UA/OHCHR_eighth_report_ on_Ukraine.pdf

UNHCR - Office of the United Nations High Commissioner for Human Rights (2016): Report on the human rights situation in Ukraine, 16 May to 15 August 2016, New York: United Nations, http:// www.ohchr.org/Documents/Countries/UA/Ukraine15thReport. pdf

UNHCR - Office of the United Nations High Commissioner for Human Rights (2017a): Report on the Human Rights Situation in Ukraine, 16 February to 15 May 2017, New York: United Nations, http://www.ohchr.org/Documents/Countries/UA/ UAReport18th_EN.pdf.

UNHCR - Office of the United Nations High Commissioner for Human Rights (2017b): Report on the Human Rights Situation in Ukraine, 16 May-15 August 2017, New York: United Nations, http://www.ohchr.org/Documents/Countries/UA/ UAReport19th_EN.pdf.

UNHCR - Office of the United Nations High Commissioner for Human Rights (2017c): Report on the Human Rights Situation in Ukraine, 16 August to 15 November 2017. New York: United Nations, https://www.ohchr.org/Documents/Countries/UA/ UAReport20th_EN.pdf.

World Bank (2015): Ukraine: Recovery and Peacebuilding Assessment, Washington, D.C.: World Bank, http://documents. worldbank.org/curated/en/879011468188335375/ pdf/96487-REPLACEMENT-FILE-WP-v1-Box391453B-PUBLICRPA-A4-Vol1-Eng-Web.pdf. 